\title{
Leavitt path algebras with at most countably many irreducible representations
}

\author{
Pere Ara and Kulumani M. Rangaswamy
}

\begin{abstract}
Let $E$ be an arbitrary directed graph with no restrictions on the number of vertices and edges and let $K$ be any field. We give necessary and sufficient conditions for the Leavitt path algebra $L_{K}(E)$ to be of countable irreducible representation type, that is, we determine when $L_{K}(E)$ has at most countably many distinct isomorphism classes of simple left $L_{K}(E)$-modules. It is also shown that $L_{K}(E)$ has finitely many isomorphism classes of simple left modules if and only if $L_{K}(E)$ is a semiartinian von Neumann regular ring with finitely many ideals. Equivalent conditions on the graph $E$ are also given. Examples are constructed showing that for each (finite or infinite) cardinal $\kappa$ there exists a Leavitt path algebra $L_{K}(E)$ having exactly $\kappa$ distinct isomorphism classes of simple right modules.
\end{abstract}

\section{Introduction}

The notion of Leavitt path algebras was introduced and initially studied in [1] and [7] as algebraic analogues of graph $\mathrm{C}^{*}$-algebras and the study of their various ring-theoretic properties has been the subject of a series of papers in recent years (see, e.g., [1]-[9], [14], and [17]). In [12], Goncalves and Royer indicated a method of constructing various representations of a Leavitt path algebra $L_{K}(E)$ over a graph $E$ by using the concept of algebraic branching systems. Expanding this, Chen [11] studied special types of irreducible representations of $L_{K}(E)$ by using the sinks as well as the infinite paths which are not tail-equivalent in the graph $E$ and he noted that these can also be considered as algebraic branching systems. For additional ways of constructing irreducible representations of $L_{K}(E)$ see [8].

In this paper we investigate the Leavitt path algebras $L_{K}(E)$ which are of countable irreducible representation type, that is, $L_{K}(E)$ having at most countably many distinct isomorphism classes of simple left/right $L_{K}(E)$-modules. For short, we call a Leavitt path algebra with this property as CIRT. In many of the past

Mathematics Subject Classification (2010): Primary 16S10; Secondary 16E50.

Keywords: Leavitt path algebra, irreducible representation, socle, von Neumann regular. 
investigations describing various algebraic properties of a Leavitt path algebra $L_{K}(E)$, the individual nature of the field $K$ did not seem to play any role at all. In this context, it may be of of some interest to note that, when $L_{K}(E)$ is CIRT and the graph $E$ contains cycles, $K$ must necessarily be a countable field. Our structure theorem states that $L_{K}(E)$ is CIRT if and only if it is the union of a smooth ascending chain of countable length consisting of graded ideals

$$
0<I_{1}<\cdots<I_{\alpha}<I_{\alpha+1}<\cdots \quad(\alpha<\tau)
$$

where $\tau$ is a countable ordinal and, for each $0 \leq \alpha<\tau, I_{\alpha+1} / I_{\alpha}$ is a direct sum of at most countably many matrix rings over $K$ and/or $K\left[x, x^{-1}\right]$. Moreover, the field $K$ is countable whenever the graph $E$ contains cycles (equivalently, $K\left[x, x^{-1}\right]$ occurs as a direct factor in $I_{\alpha+1} / I_{\alpha}$ for some $\alpha$ ).

We also show that $L_{K}(E)$ will have at most finitely many non-isomorphic simple left/right $L_{K}(E)$-modules if and only if $L_{K}(E)$ is a semi-artinian von Neumann regular ring with at most finitely many ideals. In particular, when $E$ is a finite graph, then $L_{K}(E)$ has this property exactly when $L_{K}(E)$ is an artinian semisimple ring (equivalently, the graph $E$ is acyclic). We also construct, for each arbitrary (finite or infinite) cardinal $\kappa$, a Leavitt path algebra for which the cardinality of the distinct isomorphism classes of simple right modules is exactly $\kappa$.

Acknowledgements. We are deeply grateful to the referee whose insightful comments, questions and suggestions lead to a substantial improvement of the previous version of this paper.

\section{Preliminaries}

For the general notation, terminology and results on Leavitt path algebras, we refer the reader to [1], [3] and [17]. We just give a short outline of some of the needed concepts. A (directed) graph $E=\left(E^{0}, E^{1}, r, s\right)$ consists of two sets $E^{0}$ and $E^{1}$ together with maps $r, s: E^{1} \rightarrow E^{0}$. All the graphs $E$ that we consider here are arbitrary with no restrictions on the number of vertices and the number of edges emitted by a vertex. Also $K$ stands for an arbitrary field. For each $e \in E^{1}$, we call $e^{*}$ a ghost edge. We let $r\left(e^{*}\right)$ denote $s(e)$, and we let $s\left(e^{*}\right)$ denote $r(e)$. The set of all vertices on the path $\mu$ is denoted by $\mu^{0}$. A vertex $v$ in $E$ is said to be regular if $0<\left|s^{-1}(v)\right|<\infty$. A singular vertex is a vertex which is not regular.

Given an arbitrary graph $E$ and a field $K$, the Leavitt path $K$-algebra $L_{K}(E)$ is defined to be the $K$-algebra generated by a set $\left\{v: v \in E^{0}\right\}$ of pairwise orthogonal idempotents together with a set of variables $\left\{e, e^{*}: e \in E^{1}\right\}$ which satisfy the following conditions:

(1) $s(e) e=e=e r(e)$ for all $e \in E^{1}$;

(2) $r(e) e^{*}=e^{*}=e^{*} s(e)$ for all $e \in E^{1}$;

(3) (The "CK-1 relations") For all $e, f \in E^{1}, e^{*} e=r(e)$ and $e^{*} f=0$ if $e \neq f$. 
(4) (The "CK-2 relations") For every regular vertex $v \in E^{0}$,

$$
v=\sum_{e \in E^{1}, s(e)=v} e e^{*} .
$$

A path $\mu=e_{1} \ldots e_{n}$ in $E$ is closed if $r\left(e_{n}\right)=s\left(e_{1}\right)$, in which case $\mu$ is said to be based at the vertex $s\left(e_{1}\right)$. The closed path $\mu$ is called a cycle if $s\left(e_{i}\right) \neq s\left(e_{j}\right)$ for every $i \neq j$. A graph $E$ is said to satisfy condition $(K)$ provided no vertex $v \in E^{0}$ is the base of precisely one simple closed path, i.e., either no simple closed path is based at $v$, or at least two are based at $v$. An exit for a path $\mu=e_{1} \ldots e_{n}$ is an edge $e$ such that $s(e)=s\left(e_{i}\right)$ for some $i$ and $e \neq e_{i}$.

If there is a path from vertex $u$ to a vertex $v$, we write $u \geq v$. A subset $D$ of vertices is said to be downward directed if for any $u, v \in D$, there exists a $w \in D$ such that $u \geq w$ and $v \geq w$. A subset $H$ of $E^{0}$ is called hereditary if, whenever $v \in H$ and $w \in E^{0}$ satisfy $v \geq w$, then $w \in H$. A hereditary set is saturated if, for any regular vertex $v, r\left(s^{-1}(v)\right) \subseteq H$ implies $v \in H$.

For any vertex $v$, the tree of $v$ is $T(v)=\{w: v \geq w\}$. We say there is a bifurcation at a vertex $v$, if $v$ emits more than one edge. In a graph $E$, a vertex $v$ is called a line point if there is no bifurcation or a cycle based at any vertex in $T(v)$. Thus, if $v$ is a line point, there will be a single finite or infinite line segment $\mu$ starting at $v(\mu$ could just be $v$ ) and any other path $\alpha$ with $s(\alpha)=v$ will just be an initial sub-segment of $\mu$. It was shown in [9] that $v$ is a line point in $E$ if and only if $v L_{K}(E)$ (and likewise $L_{K}(E) v$ ) is a simple right (left) ideal. Moreover, the ideal generated by all the line points in $E$ is the socle of $L_{K}(E)$. If $v$ is a line point, then it is clear that any $w \in T(v)$ is also a line point.

We shall be using the following concepts and results from [17] in our investigation. Although it is assumed in [17] that all the graphs are countable, i.e. have at most countably many vertices and edges, an examination of the proofs shows that this assumption is not needed at all. A breaking vertex of a hereditary saturated subset $H$ is an infinite emitter $w \in E^{0} \backslash H$ (that is, $s^{-1}(w)$ is an infinite set) with the property that $1 \leq\left|s^{-1}(w) \cap r^{-1}\left(E^{0} \backslash H\right)\right|<\infty$. The set of all breaking vertices of $H$ is denoted by $B_{H}$. For any $v \in B_{H}, v^{H}$ denotes the element $v-\sum_{s(e)=v, r(e) \notin H} e e^{*}$. Given a hereditary saturated subset $H$ and a subset $S \subseteq B_{H},(H, S)$ is called an admissible pair. Given an admissible pair $(H, S)$, $I_{(H, S)}$ denotes the ideal generated by $H \cup\left\{v^{H}: v \in S\right\}$. It was shown in [17] that the graded ideals of $L_{K}(E)$ are precisely the ideals of the form $I_{(H, S)}$ for some admissible pair $(H, S)$. Moreover, $L_{K}(E) / I_{(H, S)} \cong L_{K}(E \backslash(H, S))$. Here $E \backslash(H, S)$ is the quotient graph of $E$ in which $(E \backslash(H, S))^{0}=\left(E^{0} \backslash H\right) \cup\left\{v^{\prime}: v \in B_{H} \backslash S\right\}$ and $(E \backslash(H, S))^{1}=\left\{e \in E^{1}: r(e) \notin H\right\} \cup\left\{e^{\prime}: e \in E^{1}, r(e) \in B_{H} \backslash S\right\}$ and $r, s$ are extended to $(E \backslash(H, S))^{0}$ by setting $s\left(e^{\prime}\right)=s(e)$ and $r\left(e^{\prime}\right)=r(e)^{\prime}$.

If $p=e_{1} e_{2} \cdots e_{n} \cdots$ is an infinite path where the $e_{i}$ are edges, then for any positive integer $n$, let $\tau_{\leq n}(p)=e_{1} e_{2} \cdots e_{n}$ and $\tau_{>n}(p)=e_{n+1} e_{n+2} \cdots$. Two infinite paths $p$ and $q$ are said to be tail equivalent, in symbols, $p \sim q$, if there exist positive integers $m$ and $n$ such that $\tau_{>n}(p)=\tau_{>m}(q)$. Then $\sim$ is an equivalence relation. Given an equivalence class of infinite paths $[p]$, Chen [11] defines an $L_{K}(E)$-module 
action on the linear span $V_{[p]}$ of the equivalence class $[p]$ of $p$ and shows that $V_{[p]}$ becomes a simple left $L_{K}(E)$-module. He further shows that, for infinite paths $p$ and $q, V_{[p]} \cong V_{[q]}$ if and only if $p \sim q$.

\section{Leavitt path algebras of countable irreducible representa- tion type}

Throughout this and the following sections, $L$ will denote the Leavitt path algebra $L_{K}(E)$. In this section, we give a complete description of the Leavitt path algebra $L$ which has at most countably many distinct isomorphism classes of simple left/right $L$-modules. For short, we call a Leavitt path algebra with this property as CIRT.

The proof of our main theorem is derived from the following series of Propositions containing the necessary conditions for $L$ to be CIRT.

Proposition 3.1. If $L$ is CIRT, then distinct cycles in $E$ must be disjoint.

Proof. We may assume that $E$ contains cycles, since there is nothing to prove if $E$ is acyclic. Suppose that there are two different cycles $g, h$ based at the same vertex $v$. Consider the infinite path $g g g \cdots$ which, for convenience, we write as $p=g_{1} g_{2} g_{3} \cdots$ indexed by the set $\mathbf{P}$ of positive integers where $g_{i}=g$ for all $i$. Now for every subset $S$ of $\mathbf{P}$, define an infinite path $p_{S}$ by replacing $g_{i}$ by $h$ if and only if $i \in S$. Observe that this gives an uncountable family of pairwise different infinite paths on $E$. Denote by $Y$ this family of infinite paths. Now, if $q=\beta_{1} \beta_{2} \cdots$ is a path in $Y$, then the paths in $Y$ which are tail-equivalent to $q$ have the form $\gamma_{1} \cdots \gamma_{j} \beta_{i+1} \beta_{i+2} \beta_{i+3} \cdots$, where $i, j$ are non-negative integers and $\gamma_{1}, \ldots, \gamma_{j} \in\{g, h\}$. This is a countable family and so each tail-equivalence class contains countably many infinite paths in $Y$. Consequently, there are uncountably many distinct tail equivalence classes of paths in $Y$. By Chen [11], $L$ then has uncountably many non-isomorphic simple left/right $L$-modules. This contradicts the fact that $L$ is a CIRT. Hence no two cycles in $E$ have a common vertex.

Proposition 3.2. If the graph $E$ contains cycles and $L$ is CIRT, then the field $K$ must be countable.

Proof. Consider a cycle $c$ based at a vertex $v$ in $E$. Then $H=\left\{u \in E^{0}: u \supsetneqq v\right\}$ is a hereditary saturated set and $E^{0} \backslash H=M(v)$ is downward directed. By Proposition 3.1, distinct cycles have no common vertex. Hence $c$ is a cycle without exits in $M(v)$. By Theorems 3.12 and 4.3 of [14], each irreducible polynomial $g(x) \in K\left[x, x^{-1}\right]$ then gives rise to a primitive ideal $P_{g}$ of $L$, generated by $I_{\left(H, B_{H}\right)} \cup\{g(c)\}$ (and a corresponding simple module having $P_{g}$ as its annihilator). Since $L$ is CIRT, $K\left[x, x^{-1}\right]$ cannot have uncountably many irreducible polynomials. This implies that $K$ must be a countable field.

We next establish an important property for $E$ when $L$ is CIRT.

Proposition 3.3. If $L$ is CIRT, then the graph $E$ contains line points or cycles without exits, or both. 
Proof. Suppose, by way of contradiction, the graph $E$ contains no line points and no cycles without exits.

Let $v$ be a vertex in $E$, and set $F=T(v)$, seen as a subgraph of $E$, that is, we consider the tree of $v$ together with all edges in $E$ connecting the vertices of $T(v)$. It is enough to show that $F$ has uncountably many tail-equivalence classes of infinite paths. We wish to build a countable complete subgraph $G$ of $F$ having the same properties.

First, we construct an increasing family of countable complete subgraphs $F_{n}$ starting with $F_{0}=\{v\}$. Indeed, the graphs $F_{n} \subseteq F$ will satisfy the property that, for $u \in\left(F_{n}\right)^{0}$, if $u$ is a regular vertex in $F_{n}$, then $s_{F_{n}}^{-1}(u)=s_{E}^{-1}(u)$. This clearly implies that $F_{n}$ is a complete subgraph of $E$, see Definition 1.6.7 in [3]. Now assume that $F_{n}$ has been built for some $n \geq 0$. For each vertex $u \in\left(F_{n}\right)^{0}$ which is a sink in $F_{n}$ and which is a regular vertex in $E$, add to $F_{n}$ all the finitely many edges emitted by $u$ in $E$ and the end vertices of these edges. For each vertex $u \in\left(F_{n}\right)^{0}$ which is a sink in $F_{n}$ and which is an infinite emitter in $E$, add a countable number of edges emitted by $u$ in $E$ and the corresponding end vertices of these edges. This way we get the new subgraph $F_{n+1}$. Let $G$ be the union of all the $F_{n}$ 's. Then $G$ is a complete subgraph of $F$, and $G$ contains no line points and no cycles without exits.

Clearly $L_{K}(G)$ is a subalgebra of $L_{K}(F)$ and the map sending an infinite path in $G$ to the same infinite path in $F$ induces an injective map from the set $T_{G}$ of the tail-equivalence classes of infinite paths in $G$ to the set $T_{F}$ of the corresponding tail-equivalence classes of infinite paths in $F$. Thus if $T_{G}$ is uncountable so is $T_{F}$.

To show that the set $T_{G}$ is uncountable, let $X$ be the set of all infinite paths in $G$ together with the finite paths in $G$ ending in a singular vertex. For each finite path $\gamma$ (which could also be a vertex), define

$$
\mathcal{Z}(\gamma)=\left\{p \in X \text { such that } p=\gamma p^{\prime} \text {, where } p^{\prime} \text { is some path in } G\right\} .
$$

Then $X$ can be made a locally compact Hausdorff space, and the collection

$$
\left\{\mathcal{Z}(\gamma) \backslash \bigcup_{e \in \mathcal{F}} \mathcal{Z}(\gamma e)\right\}
$$

for $\gamma$ a finite path and $\mathcal{F}$ a finite set of edges starting at $r(\gamma)$, is a basis of compact open sets in $X$ (see Theorem 2.1 in [18]). Since $G$ is a countable graph, these sets form a countable basis of open and closed (compact) sets for $X$ and hence $X$ is second countable. Since $G$ does not have line points or cycles without exits, we see that $X$ has no isolated points.

Now $\mathcal{Z}(v)$ is a compact Hausdorff totally disconnected space with no isolated points and hence is isomorphic to the Cantor space (see Theorem 13 in [16]). This means $\mathcal{Z}(v)$ contains $2^{\aleph_{0}}$ infinite paths. Since $G$ is countable, an argument similar to the one used in the proof of Proposition 3.1 shows that the tail-equivalence classes of paths in $\mathcal{Z}(v)$ are at most countable. Since $|\mathcal{Z}(v)|=2^{\aleph_{0}}$, we conclude that $G$ (and hence $E$ ) contains $2^{\aleph_{0}}$ infinite paths which are not mutually tailequivalent. By Chen [11], $L$ will then have $2^{\aleph_{0}}$ non-isomorphic simple left/right $L$-modules, a contradiction.

Hence $E$ always contains line points and/or cycles without exits. 
For a ring $R$ and any index set $\Lambda$, we denote by $M_{\Lambda}(R)$ the ring of matrices with coefficients in $R$, indexed on $\Lambda$, and having only finitely many nonzero entries.

We are now ready to prove the main result of this section.

Theorem 3.4. Let $E$ be an arbitrary graph and $K$ be any field. Then the following properties are equivalent:

(i) $L=L_{K}(E)$ is CIRT;

(ii) $L$ is the union of a well-ordered smooth ascending chain of countable length consisting of graded ideals

$$
0<I_{1}<\cdots<I_{\alpha}<I_{\alpha+1}<\cdots \quad(\alpha<\tau)
$$

where, $\tau$ is a countable ordinal, for each $0 \leq \alpha<\tau, I_{\alpha+1} / I_{\alpha}$ is a direct sum of at most countably many matrix rings $M_{\Lambda}(R)$, where $\Lambda$ are arbitrarily-sized index sets and $R$ is either $K$ or $K\left[x, x^{-1}\right]$. Moreover, $K$ will be a countable field whenever $E$ contains cycles.

Proof. Assume (i). By Proposition 3.3, the graph $E$ contains line points and/or cycles without exits. Let $I_{1}$ be the ideal generated by all the line points and vertices on all the cycles without exits in $E$. Then $I_{1}=I(H, \emptyset)$, where $H=I_{1} \cap E^{0}$. By [3], $I_{1}$ is a direct sum of matrices of the form $M_{n_{i}}(K)$ and/or $M_{l_{j}}\left(K\left[x, x^{-1}\right]\right)$ where $n_{i}, l_{j}$ are cardinal numbers. Suppose we have defined a graded ideal $I_{\alpha}=$ $I\left(H_{\alpha}, B_{\alpha}\right)$ for some $\alpha \geq 1$ where $H_{\alpha}=I_{\alpha} \cap E^{0}$ and $B_{\alpha}=\left\{v \in B_{H_{\alpha}}: v^{H_{\alpha}} \in I_{\alpha}\right\}$. If $I_{\alpha} \neq L$, consider $L / I_{\alpha} \cong L_{K}\left(E \backslash\left(H_{\alpha}, B_{\alpha}\right)\right)$. Now $L / I_{\alpha}$ is CIRT and hence, by Proposition 3.3, $E \backslash\left(H_{\alpha}, B_{\alpha}\right)$ contains line points and/or cycles without exits. Define the ideal $I_{\alpha+1} \supset I_{\alpha}$ so that $I_{\alpha+1} / I_{\alpha}$ is the ideal generated by all the line points and the vertices on all the cycles without exits in $E \backslash\left(H_{\alpha}, B_{\alpha}\right)$. By [3], $I_{\alpha+1} / I_{\alpha}$ is then a direct sum of matrices of the form $M_{n_{i}}(K)$ and/or $M_{l_{j}}\left(K\left[x, x^{-1}\right]\right)$ where $n_{i}, l_{j}$ are cardinal numbers. If $\gamma$ is a limit ordinal and $I_{\alpha}$ has been defined for all $\alpha<\gamma$, then define $I_{\gamma}=\bigcup_{\alpha<\gamma} I_{\alpha}$. By transfinite induction, we then obtain a smooth ascending chain $(*)$ of graded ideals with the desired properties and its union is $L$. Now each graded ideal is isomorphic to the Leavitt path algebra of a suitable graph (see [15]) and so is a ring with local units. Hence every left/right ideal of $I_{\alpha+1}$ is also a left/right ideal of $L$. It is then readily seen that every simple $I_{\alpha+1}$-module is isomorphic to a simple $L$-module. Since $L$ is CIRT, the length of the chain $\tau$ is countable and that, for each $\alpha<\tau, I_{\alpha+1} / I_{\alpha}$ is a direct sum of at most countably many matrices of the form $M_{n_{i}}(K)$ and/or $M_{l_{j}}\left(K\left[x, x^{-1}\right]\right)$.

Moreover, Proposition 3.2 shows that the field $K$ must be countable if $E$ contains cycles. This proves (ii).

Assume (ii), so that $L$ is the union of a chain of graded ideals satisfying the stated properties. Let $S=L / M$ be a simple module where $M$ is a maximal left ideal of $L$. Let $\beta$ be the smallest ordinal such that $I_{\beta} \nsubseteq M$. Clearly $\beta$ is a non-limit ordinal. Let $\beta=\alpha+1$. Then $I_{\alpha} \subseteq M$. So,

$$
S=\left(M+I_{\alpha+1}\right) / M \cong I_{\alpha+1} /\left(I_{\alpha+1} \cap M\right) \cong\left(I_{\alpha+1} / I_{\alpha}\right) /\left[\left(I_{\alpha+1} \cap M\right) / I_{\alpha}\right] .
$$


Thus every simple left $L$-module is isomorphic to a simple left module over the ring $I_{\alpha+1} / I_{\alpha}$ for some $\alpha$ with $0 \leq \alpha<\tau$. Now for each $\alpha, I_{\alpha+1} / I_{\alpha}$ is CIRT, due to the fact that $I_{\alpha+1} / I_{\alpha}$ is a direct sum of at most countably many matrix rings $M_{n_{k}}(K)$ and matrix rings $M_{r_{j}}\left(K\left[x, x^{-1}\right]\right)$, where $n_{k}, r_{j}$ are arbitrarily-sized cardinal numbers and that $K$ is countable whenever $r_{j} \neq 0$ for some $r_{j}$. Since $\tau$ is a countable ordinal, it is readily seen that $L$ is also CIRT.

Remark 3.5. In Theorem 3.4, if the graph $E$ is acyclic, then for every $\alpha<\tau$, $I_{\alpha+1} / I_{\alpha}$ is isomorphic to a direct sum of matrix rings over $K$ and hence is a direct sum of simple modules. Thus the chain $(*)$ becomes the socular chain for $L$. In other words, $L$ becomes a semi-artinian von Neumann regular ring with countable Loewy length. Note that, in this case, there is no restriction on the cardinality of the field $K$.

\section{Leavitt path algebras of finite irreducible representation type}

We now wish to specialize to the case when the Leavitt path algebra $L=L_{K}(E)$ is of finite irreducible representation type, that is, when $L$ has at most finitely many distinct isomorphism classes of simple left $L$-modules. In this case, $L$ satisfies conditions stronger than those of Theorem 3.4. Specifically, $L$ becomes a semiartinian von Neumann regular ring with finitely many two-sided ideals. Moreover, the result holds for arbitrary fields $K$ without any restrictions.

We begin with the following lemma, and its corollary, which are used in the proof of Theorem 4.3.

Lemma 4.1. Suppose $R$ is a von Neumann regular ring and $A$ is a non-zero proper ideal of $R$. Then every simple left $R$-module is isomorphic to a simple left module over $R / A$ or $A$. Conversely, every simple left module over $R / A$, or $A$ is isomorphic to some simple left $R$-module.

Proof. Let $R / M$ be a simple left $R$-module, where $M$ is a maximal left ideal of $R$. Then it is easy to see that $R / M$ is isomorphic to a simple left module over $R / A$ or $A$ according as (i) $M \supset A$, or (ii) $M \nsupseteq A$.

To prove the converse, we only consider the case of a simple left $A$-module $S$, as the other case is trivial. Write $S=A / N$, where $N$ is a maximal left ideal of $A$. For $n \in N$ there is an idempotent $e$ in $A$ such that $e n=n$. It follows from this that $N$ is indeed a left $R$-module, and so $S=A / N$ is a simple $R$-module.

Corollary 4.2. Suppose $L_{K}(E)$ is semi-artinian ring with finitely many ideals. Then $L_{K}(E)$ has at most finitely many non-isomorphic simple left modules.

Proof. Suppose the semi-artinian $\operatorname{ring} L=L_{K}(E)$ has only finitely many two-sided ideals. Then we can build a finite ascending chain of two-sided ideals $0<S_{1}<$ $\cdots<S_{n}=L$ where, for each $i=1, \ldots, n-1, S_{i+1} / S_{i}$ is a simple ring which, being also semi-artinian, is a direct sum of isomorphic simple left ideals. Consequently, 
each $S_{i+1} / S_{i}$ has a single isomorphism class of simple left $S_{i+1} / S_{i}$-modules. Also by [6], $L_{K}(E)$ is von Neumann regular. We then conclude, by Lemma 4.1 , that $L$ has exactly $n$ distinct isomorphism classes of simple left $L$-modules.

Theorem 4.3. Let $E$ be an arbitrary graph and $K$ be any field. Then the following are equivalent for the Leavitt path algebra $L=L_{K}(E)$ :

(i) L has at most finitely many non-isomorphic simple left/right L-modules;

(ii) $L$ is a semi-artinian ring with finitely many two-sided ideals.

Proof. Assume (i). Since there are only finitely many non-isomorphic simple left modules, $L_{K}(E)$ has only finitely many distinct primitive ideals. We wish to show that there are no non-graded prime ideals in $L_{K}(E)$. Now any non-graded prime ideal of $L_{K}(E)$ is primitive (see Theorem 4.3 in [14]). From Theorem 3.12 of [14], the non-graded prime ideals of $L_{K}(E)$ are precisely the ideals $P$ generated by $I_{(H, S)} \cup\{f(c)\}$, where $P \cap E^{0}=H, S=\left\{v \in B_{H}: v^{H} \in P\right\}, c$ is a cycle without exits in $E \backslash H$, and $f$ is an irreducible polynomial in $K\left[x, x^{-1}\right]$. So, if $L_{K}(E)$ has one such non-graded prime ideal $P$, then corresponding to each of the infinitely many irreducible polynomials $g$ in $K\left[x, x^{-1}\right], L_{K}(E)$ will have a non-graded prime (and hence a primitive) ideal $P_{g}$ generated by $I_{(H, S)} \cup\{g(c)\}$. This contradicts that $L_{K}(E)$ has only a finite number of primitive ideals. Thus every prime ideal of $L_{K}(E)$ must be graded and we appeal to Corollary 3.13 in [14] to conclude that the graph $E$ satisfies condition (K). From Theorem 6.16 in [17], we then conclude that every ideal of $L_{K}(E)$ is graded.

Thus if $I$ is an ideal of $L_{K}(E)$ then, being a graded ideal, $I=I_{(H, S)}$, where $H=I \cap E^{0}$ and $S=\left\{v \in B_{H}: v^{H} \in I\right\}$ and, moreover, $L_{K}(E) / I \cong L_{K}(E \backslash(H, S))$ by [17]. As the Jacobson radical of the Leavitt path algebra $L_{K}(E \backslash(H, S))$ is zero (see [6]), we conclude that $I$ is the intersection of all the primitive ideals containing $I$. As there are only finitely many primitive ideals in $L_{K}(E)$, we then conclude that $L_{K}(E)$ contains only finitely many distinct ideals which are all of the form $I_{(H, S)}$ for some admissible pair $(H, S)$.

We also claim that $E$ contains no cycles. Because if there is a cycle $g$ in $E$, then by condition $(\mathrm{K})$ there will be another cycle $h \neq g$ sharing a common vertex with $g$ and this contradicts Proposition 3.1. Hence $E$ is acyclic and $L$ is von Neumann regular, by [5]. Also, by Proposition 3.3, $E$ contains line points and so $S_{1}=\operatorname{Soc}(L) \neq 0$. Also, since there are only finitely many ideals, $S_{1}$ is a direct sum of finitely many homogeneous components: $S_{1}=\bigoplus_{i=1}^{n_{1}} S_{1 i}$ with $S_{1 i}$ a direct sum of isomorphic simple left ideals of $L$. Let $H=S_{1} \cap E^{0}$. Now $L / S_{1} \cong L_{K}(E \backslash(H, \emptyset))$ satisfies the same hypothesis as $L$ and hence has a nonzero socle $S_{2} / S_{1}$. Proceeding like this and using the fact that $L$ has at most finitely many ideals, we conclude $L$ is the union of a finite ascending chain of ideals $\{0\}=S_{0} \subset S_{1} \subset \cdots \subset S_{m}=L$ where, for each $i, S_{i+1} / S_{i}=\operatorname{Soc}\left(L / S_{i}\right)$ and is a direct sum of finitely many homogeneous components. This proves (ii).

(ii) $\Longrightarrow($ i). This follows from Corollary 4.2 .

Remark 4.4. One might wonder what are the finite lattices appearing as ideal lattices of the algebras $L_{K}(E)$ characterized in Theorem 4.3. Indeed, observe that 
the lattice of ideals of a von Neumann regular ring is a distributive lattice. It follows from Bergman's theorem, [10], that every finite distributive lattice can be represented as the lattice of ideals of a unital ultramatricial algebra. On the other hand, Proposition 2.12 in [13] asserts that this unital ultramatricial algebra is Morita-equivalent to a Leavitt path algebra $L_{K}(E)$ of an acyclic graph. For this graph $E$, the conditions in Theorem 4.3 (ii) necessarily hold. In conclusion, we see that the class of lattices appearing as ideal lattices of the algebras described in Theorem 4.3 is exactly the class of all finite distributive lattices.

\section{Equivalent graphical conditions}

In this section we describe the graphical properties of $E$ under which the Leavitt path algebra $L_{K}(E)$ is of finite irreducible representation type. We begin with a simple lemma describing when two line points generate isomorphic simple right ideals.

Lemma 5.1. Given two line points $u, v, u L_{K}(E) \cong v L_{K}(E)$ if and only if $T(u) \cap$ $T(v)$ is not empty.

Proof. Suppose $\theta: u L_{K}(E) \rightarrow v L_{K}(E)$ is an isomorphism. Then $\theta$ is given by the left multiplication by the non-zero element $\theta(u)=v s u$ for some $s \in L_{K}(E)$. We can clearly assume that $s=v s u$. Write $s=\sum_{i=1}^{m} k_{i} \alpha_{i} \beta_{i}^{*}$ where $k_{i} \in K$ and $\alpha_{i}, \beta_{i}$ are finite paths in $E$. If a term $k_{i} \alpha_{i} \beta_{i}^{*}=k_{i} v \alpha_{i} \beta_{i}^{*} u \neq 0$, then $v=s\left(\alpha_{i}\right)$, $u=s\left(\beta_{i}\right)$ and $r\left(\alpha_{i}\right)=r\left(\beta_{i}\right)=w$, so $w \in T(u) \cap T(v)$. Conversely, suppose $w \in T(u) \cap T(v)$. Since $u$ is a line point, so is $w$ and there is a unique path $\mu$ from $u$ to $w$. Then $u a \longmapsto w \mu^{*} u a$ is an isomorphism from the simple module $u L_{K}(E)$ to $w L_{K}(E)$ with the map $w b \longmapsto u \mu w b$ being the inverse isomorphism. By a similar argument, $v L_{K}(E) \cong w L_{K}(E)$. Consequently, $u L_{K}(E) \cong v L_{K}(E)$.

The next theorem describes the graphical conditions on $E$ under which $L_{K}(E)$ is of finite irreducible representation type.

Theorem 5.2. Let $E$ be an arbitrary graph and let $K$ be any field. Then the Leavitt path algebra $L_{K}(E)$ is of finite irreducible representation type if and only if all of the following conditions hold:

(i) E is acyclic;

(ii) $E^{0}$ has only finitely many distinct hereditary saturated subsets $H$ and for each such $H$ the corresponding set $B_{H}$ of breaking vertices is finite; and

(iii) In the poset of admissible pairs in $E,\left(E^{0}, \emptyset\right)$ is the supremum of a finite ascending chain

$$
\begin{aligned}
\left(H_{0}=\emptyset, \emptyset\right) & <\left(H_{1}, \emptyset\right)<\left(H_{1}, B_{H_{1}}\right)<\left(H_{2}, B_{H_{1}} \cap B_{H_{2}}\right)<\left(H_{2}, B_{H_{2}}\right) \\
& <\left(H_{3}, B_{H_{2}} \cap B_{H_{3}}\right)<\left(H_{3}, B_{H_{3}}\right)<\cdots
\end{aligned}
$$

where, for $j \geq 0, H_{j+1}$ is a hereditary saturated subset of $E^{0}$ and $H_{j+1} \backslash H_{j}$ is the hereditary saturated closure of the set of line points in $E \backslash\left(H_{j}, B_{H_{j}}\right)$. 
Proof. If $L_{K}(E)$ has finite irreducible representation type, then in the proof of Theorem 4.3 it was shown that $E$ is acyclic and that $L_{K}(E)$ has at most finitely many two-sided ideals (which are all graded). The latter property is equivalent to condition (ii) by Theorem 5.7 in [17]. To prove condition (iii), we shall use the fact, established in Theorem 4.3, that $L_{K}(E)$ (and each of its homomorphic images) is semi-artinian. We wish to construct a chain of ideals of the form $I_{(H, S)}$. Let $J_{1}=\operatorname{Soc}\left(L_{K}(E)\right)$. By [9], $J_{1}$ is the ideal generated by the hereditary saturated closure $H_{1}$ of the set $T_{1}$ of all the line points in $E$. Now $J_{1}=I_{\left(H_{1}, \emptyset\right)}$ and, by [17], $J_{1}$ is the kernel of an epimorphism $f: L_{K}(E) \longrightarrow L_{K}\left(E \backslash\left(H_{1}, \emptyset\right)\right)$ where $\left(E \backslash\left(H_{1}, \emptyset\right)\right)^{0}=E^{0} \backslash H_{1} \cup\left\{v^{\prime}: v \in B_{H_{1}}\right\}$ and that $f\left(v^{H_{1}}\right)=v^{\prime}$ for all $v \in B_{H_{1}}$. Let $J_{2}=I_{\left(H_{1}, B_{H_{1}}\right)}=\left\langle H_{1},\left\{v^{H_{1}}: v \in B_{H_{1}}\right\}\right\rangle$. so that $J_{2} / J_{1} \cong\left\langle\left\{v^{\prime}: v \in B_{H_{1}}\right\}\right\rangle \subset$ $L_{K}\left(E \backslash\left(H_{1}, \emptyset\right)\right)$. By [17], $L_{K}(E) / J_{2} \cong L_{K}\left(E \backslash\left(H_{1}, B_{H_{1}}\right)\right) \cong L_{K}\left(E \backslash H_{1}\right)$ since $\left(E \backslash\left(H_{1}, B_{H_{1}}\right)\right)^{0}=E^{0} \backslash H_{1}$ and $\left(E \backslash\left(H_{1}, B_{H_{1}}\right)\right)^{1}=\left\{e \in E^{1}: r(e) \notin H_{1}\right\}$. Now $L_{K}(E) / J_{2}$ has a non-zero socle (being semi-artinian) and let $H_{2}$ be a hereditary saturated subset of $E^{0}$ containing $H_{1}$ such that $H_{2} \backslash H_{1}$ is the hereditary saturated closure of the set $T_{2}$ of all the line points in $E \backslash\left(H_{1}, B_{H_{1}}\right)$. Define

$$
J_{3}=\left\langle J_{2}, H_{2}\right\rangle=\left\langle H_{2},\left\{v^{H_{1}}: v \in B_{H_{1}}\right\}\right\rangle
$$

so that

$$
J_{3} / J_{2} \cong\left\langle H_{2} \backslash H_{1}\right\rangle=\operatorname{Soc}\left(E \backslash\left(H_{1}, B_{H_{1}}\right)\right) \subset L_{K}\left(E \backslash\left(H_{1}, B_{H_{1}}\right)\right) \cong L_{K}\left(E \backslash H_{1}\right) .
$$

If $v \in B_{H_{1}} \backslash B_{H_{2}}$, then $r\left(s^{-1}(v)\right) \subset H_{2} \subset J_{3}$ and since $v^{H_{1}} \in J_{3}$, we conclude that $v \in J_{3}$. In the isomorphism $\left.L_{K}(E) / J_{2} \cong L_{K}\left(E \backslash H_{1}\right)\right)$, $v$ gets mapped to an element in $\left(J_{3} / J_{2}\right) \cap\left(E^{0} \backslash H_{1}\right)=H_{2} \backslash H_{1}$ and so $v \in H_{2}$. Thus $B_{H_{1}} \backslash B_{H_{2}} \subset H_{2}$ and so $J_{3}=\left\langle H_{2},\left\{v^{H_{2}}: v \in B_{H_{1}} \cap B_{H_{2}}\right\}\right\rangle=I_{\left(H_{2}, B_{H_{1}} \cap B_{H_{2}}\right)}$. Note that, in the isomorphism $L_{K}(E) / J_{1} \cong L_{K}\left(E \backslash\left(H_{1}, \emptyset\right)\right), J_{3} / J_{1}$ maps to $\operatorname{Soc}\left(L_{K}\left(E \backslash\left(H_{1}, \emptyset\right)\right)\right)$. Define $J_{4}=I_{\left(H_{2}, B_{H_{2}}\right)}$. Proceeding like this, we obtain a chain of ideals

$$
\begin{aligned}
\{0\}=I_{\left(H_{0}=\emptyset, \emptyset\right)} & \subset I_{\left(H_{1}, \emptyset\right)} \subset I_{\left(H_{1}, B_{H_{1}}\right)} \subset I_{\left(H_{2}, B_{H_{1}} \cap B_{H_{2}}\right)} \\
& \subset I_{\left(H_{2}, B_{H_{2}}\right)} \subset I_{\left(H_{3}, B_{H_{2}} \cap B_{H_{3}}\right)} \subset I_{\left(H_{3}, B_{H_{3}}\right)} \subset \cdots
\end{aligned}
$$

whose union is $L_{K}(E)$. Here, for each $j \geq 0, H_{j+1}$ is a hereditary saturated subset of $E^{0}$ and $H_{j+1} \backslash H_{j}$ is the hereditary saturated closure of the set $T_{j+1}$ of all the line points in $E \backslash\left(H_{j}, B_{H_{j}}\right)$. Note that, for each $j \geq 0$, there are only finitely many equivalence classes of line points in $E \backslash\left(H_{j}, B_{H_{j}}\right)$, due to condition (ii). Our construction shows that, in the poset of admissible pairs, $\left(E^{0}, \emptyset\right)$ is then the supremum of a finite ascending chain

$$
\begin{aligned}
(\emptyset, \emptyset) & <\left(H_{1}, \emptyset\right)<\left(H_{1}, B_{H_{1}}\right)<\left(H_{2}, B_{H_{1}} \cap B_{H_{2}}\right) \\
& <\left(H_{2}, B_{H_{2}}\right)<\left(H_{3}, B_{H_{2}} \cap B_{H_{3}}\right)<\left(H_{3}, B_{H_{3}}\right)<\cdots
\end{aligned}
$$

where the sets $H_{j}$ are as described above.

Conversely, condition (i) implies, by [5], that $L_{K}(E)$ is von Neumann regular and condition (ii) implies that it has only finitely many two-sided ideals (which are all graded ideals). Consider the chain indicated in condition (iii), and the 
corresponding chain of ideals $(* *)$ constructed above. Denote $I_{\left(H_{1}, \emptyset\right)}$ by $S_{1}$ and, for each $i \geq 2$, denote $I_{\left(H_{i}, B_{H_{i-1}} \cap B_{H_{i}}\right)}$ by $S_{i}$. Then we get the ascending finite chain

$$
\{0\} \subset S_{1} \subset \cdots \subset S_{t}=L_{K}(E)
$$

where $S_{1}=\operatorname{Soc}\left(L_{K}(E)\right)$ and for each $i, S_{i+1} / S_{i}=\operatorname{Soc}\left(L_{K(E)} / S_{i}\right)$. Hence $L_{K}(E)$ is a semi-artinian ring. By Theorem $4.3, L_{K}(E)$ is of finite irreducible representation type.

Example 5.3. To illustrate Theorem 5.2, consider the graph $P_{3}$ depicted in the next section: it is clearly acyclic. Its hereditary saturated subsets of vertices are: $H_{0}=\emptyset$, the empty set; $H_{1}=\left\{v_{11}, v_{12}, v_{13}, \ldots\right\} ; H_{2}=H_{1} \cup\left\{v_{21}, v_{22}, v_{23}, \ldots\right\} ;$ $H_{3}=\left(P_{3}\right)^{0}$. Since the graph is row-finite there are no breaking vertices. Then the chain in condition (iii) is given by

$$
(\emptyset, \emptyset)<\left(H_{1}, \emptyset\right)<\left(H_{2}, \emptyset\right)<\left(H_{3}=\left(P_{3}\right)^{0}, \emptyset\right) .
$$

We point out that one can build examples showing that no two of the three conditions in Theorem 5.2 imply the third.

\section{Examples}

In this section, we wish to illustrate Theorems 3.4 and 4.3 by constructing nontrivial examples of $L_{K}(E)$ which, being semi-artinian (von Neumann regular) rings, are the union of a finite or infinite socular chain

$$
0<S_{1}<S_{2}<\cdots<S_{n}<\cdots
$$

where $S_{1}=\operatorname{Soc}\left(L_{K}(E)\right)$ and, for each $j<n, S_{j+1} / S_{j}=\operatorname{Soc}\left(L_{K}(E) / S_{j}\right)$. To have a really simple example, one can assume that $S_{1}$ and each $S_{j+1} / S_{j}$ are direct sums of isomorphic simple modules. Since the socle of a Leavitt path algebra is generated by line points in the graph, the assumed property on $S_{1}$ and $S_{j+1} / S_{j}$ imply that the line points belonging to $S_{1}$ (and likewise in $S_{j+1} / S_{j}$ ) all must form a single straight line segment.

It is this idea that was used to construct the "pyramid" examples in reference [6]. In some sense these examples are the simplest examples of semi-artinian Leavitt path algebras of infinite graphs with arbitrary Loewy length.

We will show that, for each (finite or infinite) cardinal $\kappa$, the Leavitt path algebra $L_{K}\left(P_{\kappa}\right)$ of the pyramid graph $P_{\kappa}$ has exactly $\kappa$ distinct isomorphism classes of simple right $L_{K}(E)$-modules.

Let $P_{1}$ be the graph

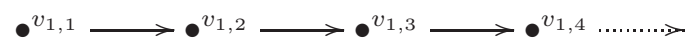

consisting of a single infinite path. Now all the vertices $v_{1, i}$ are line points in $P_{1}$ and so $v_{1, i} L_{K}\left(P_{1}\right)$ is a simple module for all $i$ (see [9]). Also $L_{K}\left(P_{1}\right)=\oplus_{i} v_{1, i} L_{K}\left(P_{1}\right)=$ 
$\operatorname{Soc}\left(L_{K}\left(P_{1}\right)\right)$. From Lemma 5.1 it is clear that, for all $i<j, v_{1, i} L_{K}\left(P_{1}\right) \cong$ $v_{1, j} L_{K}\left(P_{1}\right)$. Since $L_{K}\left(P_{1}\right)$ is a direct sum of simple modules, every simple right $L_{K}\left(P_{1}\right)$-module is isomorphic to the simple right ideal $v_{1, i} L_{K}\left(P_{1}\right)$ and we conclude that all the simple right $L_{K}\left(P_{1}\right)$-modules are isomorphic.

Let $P_{2}$ be the graph

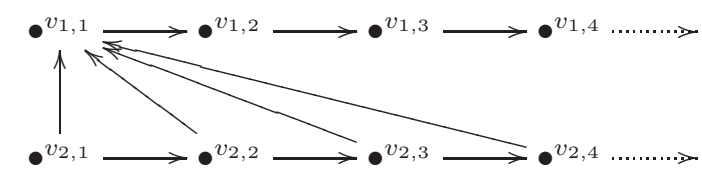

Now the line points in the graph $P_{2}$ are the vertices in the first row, namely, $v_{1,1}, v_{1,2}, v_{1,3}, \ldots$ and they generate the socle $S$ of $P_{2}$ which, from the explanation in describing $L_{K}\left(P_{1}\right)$ above, is a direct sum of isomorphic faithful simple right $L_{K}\left(P_{2}\right)$-modules. Also, by [17], $P_{2} / S \cong L_{K}(F)$ where $F \cong P_{1}$ and so $P_{2} / S=\operatorname{Soc}\left(P_{2} / S\right)$ is a direct sum of isomorphic simple modules annihilated by the ideal $S$. Thus $L_{K}\left(P_{2}\right)$ has exactly two distinct isomorphism classes of simple $L_{K}\left(P_{2}\right)$-modules.

Let $P_{3}$ be the graph

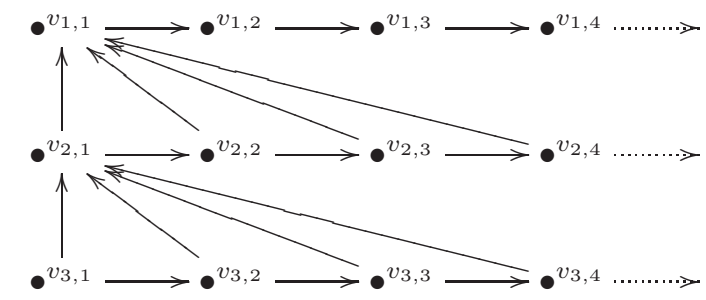

As above, the vertices $v_{1,1}, v_{1,2}, v_{1,3}, \ldots$ generate the socle $S$ of $L_{K}\left(P_{3}\right)$ which is a direct sum of isomorphic faithful simple $L_{K}\left(P_{3}\right)$-modules and $L_{K}\left(P_{3}\right) / S \cong$ $L_{K}\left(P_{2}\right)$. Clearly, by Lemma 4.1 and the description of $L_{K}\left(P_{2}\right)$ above, $S_{2} / S \cong$ $\operatorname{Soc}\left(L_{K}\left(P_{2}\right)\right)$ is a direct sum of isomorphic simple $L_{K}\left(P_{3}\right)$-modules annihilated by the ideal $S$ and that

$$
L_{K}\left(P_{3}\right) / S_{2} \cong L_{K}\left(P_{2}\right) / \operatorname{Soc}\left(L_{K}\left(P_{2}\right)\right) \cong \operatorname{Soc}\left[L_{K}\left(P_{2}\right) / \operatorname{Soc}\left(L_{K}\left(P_{2}\right)\right]\right.
$$

is a direct sum of isomorphic simple $L_{K}\left(P_{3}\right)$-modules annihilated by the ideal $S_{2}$. Thus we conclude that $L_{K}\left(P_{3}\right)$ has exactly three distinct isomorphism classes of simple right $L_{K}\left(P_{3}\right)$-modules.

Proceeding like this, we conclude, by simple induction, that for any positive integer $n$, the Leavitt path algebra $L_{K}\left(P_{n}\right)$ of the "pyramid" graph $P_{n}$ with $n$ "layers" has exactly $n$ distinct isomorphism classes of simple right $L_{K}\left(P_{n}\right)$-modules.

Let $P_{\omega}=\bigcup_{n \in \mathbb{N}} P_{n}$ be the "pyramid" graph of length $\omega$ constructed inductively and represented pictorially as follows. 


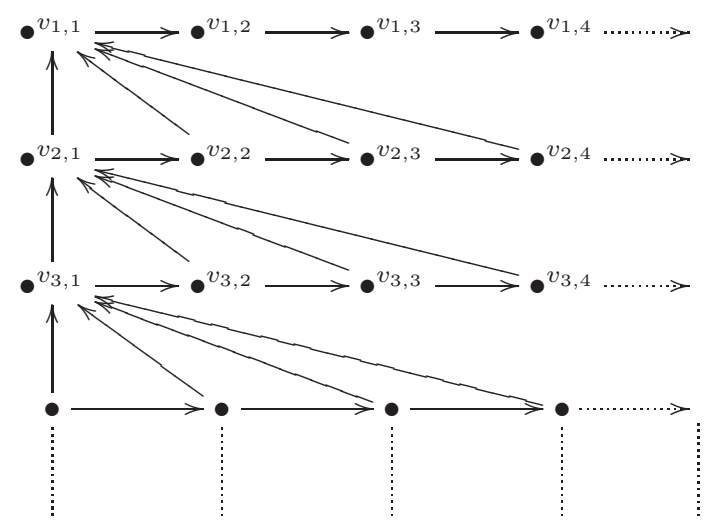

Again, by induction, it follows that $L_{K}\left(P_{\omega}\right)$ has exactly $\omega$ distinct isomorphism classes of simple $L_{K}\left(P_{\omega}\right)$-modules.

The graph $P_{\omega+1}$ is obtained from the graph $P_{\omega}$ by adding a single vertex $v_{\omega+1}$ and connecting it by an edge to each of the vertices $v_{j, 1}$ for $j<\omega$ in the graph $P_{\omega}$. Specifically, $\left(P_{\omega+1}\right)^{0}=\left(P_{\omega}\right)^{0} \cup\left\{v_{\omega+1}\right\},\left(P_{\omega+1}\right)^{1}=\left(P_{\omega}\right)^{1} \cup\left\{e_{\omega+1, j}: j<\omega\right\}$ where, for each $j, s\left(e_{\omega+1, j}\right)=v_{\omega+1}$ and $r\left(e_{\omega+1, j}\right)=v_{j, 1}$. If $S_{\omega}$ denotes the $\omega$-socle being the ideal generated by all the vertices in $P_{\omega}$, then $L_{K}\left(P_{\omega+1}\right) / S_{\omega}$ is a simple $L_{K}\left(P_{\omega+1}\right)$-module whose annihilator ideal is $S_{\omega}$ and it is isomorphic to the Leavitt path algebra of a graph $\left\{{ }^{v_{\omega+1}} \bullet\right.$ consisting of a single vertex and no edges. Clearly $L_{K}\left(P_{\omega+1}\right)$ has $\omega+1$ distinct isomorphism classes of simple $L_{K}\left(P_{\omega+1}\right)$-modules.

Proceeding this way, as was shown in [6], we can construct, by transfinite induction, a "pyramid" graph $P_{\lambda}$ for each ordinal $\lambda$. The Leavitt path algebra $L_{K}\left(P_{\lambda}\right)$ is a semi-artinian von Neumann ring of Loewy length $\lambda$ such that, for each $\alpha<\lambda$, the quotient $S_{\alpha+1} / S_{\alpha}$ of successive socles is isomorphic to the Leavitt path algebra of an infinite line segment (like the graph $P_{1}$ ) or a graph $\{\bullet\}$ consisting of a single vertex and no edges, according as $\alpha$ is a successor or a limit ordinal. Thus $S_{\alpha+1} / S_{\alpha}$ has exactly one isomorphism class of simple modules (annihilated by $\left.S_{\alpha}\right)$. By transfinite induction, one can then show that the Leavitt path algebra $L_{K}\left(P_{\lambda}\right)$ has exactly $|\lambda|$ isomorphism classes of simple $L_{K}\left(P_{\lambda}\right)$-modules.

\section{References}

[1] Abrams, G. And Aranda Pino, G.: The Leavitt path algebra of a graph. J. Algebra 293 (2005), no. 2, 319-334.

[2] Abrams, G., Aranda Pino, G. and Siles Molina, M.: Finite dimensional Leavitt path algebras. J. Pure Appl. Algebra 209 (2007), no. 3, 753-762.

[3] Abrams, G., Ara, P. And Siles Molina, M.: Leavitt path algebras - a handbook and a primer. Springer (in press).

[4] Abrams, G., Bell, J. and Rangaswamy, K. M.: On prime nonprimitive von Neumann regular algebras. Trans. Amer. Math. Soc. 366 (2014), no. 5, 2375-2392. 
[5] Abrams, G. and Rangaswamy, K. M.: Regularity conditions for arbitrary Leavitt path algebras. Algebr. Represent. Theory 13 (2010), no. 3, 319-334.

[6] Abrams, G., Rangaswamy, K. M. and Siles Molina, M.: The socle series of a Leavitt path algebra. Israel J. Math. 184 (2011), 413-435.

[7] Ara, P., Moreno, M. A. And Pardo, E.: Nonstable K-theory for graph algebras. Algebr. Represent. Theory 10 (2007), no. 2, 157-178.

[8] Ara, P. And Rangaswamy, K. M.: Finitely presented simple modules over Leavitt path algebras. J. Algebra 417 (2014), 333-352.

[9] Aranda Pino, G., Martín Barquero, D., Martín Gonzalez, C. and Siles Molina, M.: Socle theory for Leavitt path algebras of arbitrary graphs. Rev. Mat. Iberoam. 26 (2010), no. 2, 611-638.

[10] Bergman, G. M.: Von Neumann regular rings with tailor-made ideal lattices. Unpublished notes. http://math.berkeley.edu/ gbergman/papers/unpub/

[11] Chen, X. W.: Irreducible representations of Leavitt path algebras. Forum Math. 27 (2015), no. 1, 549-574.

[12] Gonchlves, D. And Royer, D.: On the representations of Leavitt path algebras. J. Algebra 333 (2011), 258-272.

[13] Raeburn, I.: Graph algebras. CBMS Regional Conference Series in Mathematics 103, American Mathematical Society, Providence, RI, 2005.

[14] Rangaswamy, K. M.: The theory of prime ideals of Leavitt path algebras over arbitrary graphs. J. Algebra 375 (2013), 73-96.

[15] Ruiz, E. And Tomforde, M.: Ideals in graph algebras. Algebr. Represent. Theory 17 (2014), no. 3, 849-861.

[16] Stone, M. H.: Applications of the theory of Boolean rings to general topology. Trans. Amer. Math. Soc. 41 (1937), no. 3, 375-481.

[17] Tomforde, M.: Uniqueness theorems and ideal structure of Leavitt path algebras. J. Algebra 318 (2007), no. 1, 270-299.

[18] Webster, S. B. G.: The path space of a directed graph. Proc. Amer. Math. Soc. 142 (2014), no. 1, 213-225.

Received November 18, 2013.

Pere Ara: Departament de Matemàtiques, Universitat Autònoma de Barcelona, 08913 Bellaterra (Barcelona), Spain.

E-mail: para@mat.uab.cat

Kulumani M. Rangaswamy: Department of Mathematics, University of Colorado at Colorado Springs, Colorado Springs, Colorado 80918, USA.

E-mail: krangasw@uccs.edu

The first-named author was supported by DGI MICIIN-FEDER MTM2011-28992-C02-01, and by the Comissionat per Universitats i Recerca de la Generalitat de Catalunya. Part of this work was done when the second-named author visited the Universitat Autonoma de Barcelona during May 2013 and he gratefully acknowledges the support and the hospitality of the faculty of the Department of Mathematics. 